\title{
A COUNTEREXAMPLE IN DYNAMICAL SYSTEMS OF THE INTERVAL
}

\author{
HSIN CHU AND XIONG JINCHENG ${ }^{1}$
}

\begin{abstract}
In [1] it was proved that if the recurrent points of a continuous map of the unit interval form a closed set, then this map has no periodic point with period not equal to a power of 2 , i.e. this map is of type $2^{\infty}$. In this paper we will construct a continuous map of the interval which is of type $2^{\infty}$ and for which the set of recurrent points is not closed. By such a counterexample it may be shown that some of the results announced in [2] are not correct.
\end{abstract}

First, recall some elementary definitions.

Suppose that $f: I \rightarrow I$ is a continuous map from the interval $I=[0,1]$ into itself. Let $f^{0}$ be the identity map on $I$. For any positive integer $n$ define $f^{n}=f \circ f^{n-1}$.

A point $x \in I$ is said to be periodic if $x=f^{m}(x)$ for some $m>0$. In this case $\min \left\{m>0: f^{m}(x)=x\right\}$ is called the period of $x$, and the finite set $\left\{f^{m}(x): m=\right.$ $1,2, \ldots\}$ is called the orbit of $x$.

A point $x \in I$ is said to be recurrent if for any neighborhood $U$ of $x, f^{m}(x) \in U$ for some $m>0$.

A subset $S$ of $I$ is said to be invariant if $f(S) \subset S$.

A continuous map of the interval is of type $2^{\infty}$ if it has no periodic point with period not equal to a power of 2 .

We will define a map $\chi_{\infty}: I \rightarrow I$ having no periodic point with period not a power of 2 such that the set $R\left(\chi_{\infty}\right)$ of recurrent points is not closed.

1. Let $\xi_{[a, b],[c, d]}$ denote the linear map from $[a, b]$ to $[c, d]$ which carries $a$ into $c$ and $b$ into $d$, and let $\bar{\xi}_{[a, b],[c, d]}$ denote the linear map from $[a, b]$ to $[c, d]$ which carries $a$ into $d$ and $b$ into $c$.

For any positive integer $k \geq 3$, let

$$
a_{k}=\frac{1}{2^{k}}, \quad b_{k}=1-\frac{3}{2^{k}}, \quad c_{k}=1-\frac{2}{2^{k}} \quad \text { and } \quad d_{k}=1-\frac{1}{2^{k}},
$$

and define a map $\mu_{k}: I \rightarrow I$ such that

$$
\begin{aligned}
\left.\mu_{k}\right|_{\left[0, a_{k}\right]} & =\xi_{\left[0, a_{k}\right],\left[0, c_{k}\right]}, \\
\left.\mu_{k}\right|_{\left[a_{k}, b_{k}\right]} & =\xi_{\left[a_{k}, b_{k}\right],\left[c_{k}, d_{k}\right]}, \\
\left.\mu_{k}\right|_{\left[b_{k}, c_{k}\right]} & =\xi_{\left[b_{k}, c_{k}\right],\left[a_{k}, d_{k}\right]}, \\
\left.\mu_{k}\right|_{\left[c_{k}, d_{k}\right]} & =\xi_{\left[c_{k}, d_{k}\right],\left[a_{k}, b_{k}\right]}, \\
\left.\mu_{k}\right|_{\left[d_{k}, 1\right]} & =\xi_{\left[d_{k}, 1\right],\left[b_{k}, 1\right]},
\end{aligned}
$$

Received by the editors October 15, 1984.

1980 Mathematics Subject Classification. Primary 58F20, 34C35.

Key words and phrases. Recurrent point, periodic point, type $2^{\infty}$

${ }^{1}$ On leave of absence from the Department of Mathematics, University of Science and Technology of China, Hefei, Anhui, People's Republic of China. 
It is easy to see that the map $\mu_{k}$ is well defined and continuous.

The following proposition is obvious.

PROPOSITION 1. The set of periodic points of $\mu_{k}$ is $\left\{0,1, e_{k}\right\} \cup\left[a_{k}, b_{k}\right] \cup\left[c_{k}, d_{k}\right]$, where $e_{k} \in\left[b_{k}, c_{k}\right]$, in which $0,1, e_{k}$ are fixed points of $\mu_{k}$ and every point in $\left[a_{k}, b_{k}\right] \cup$ $\left[c_{k}, d_{k}\right]$ has period 2 .

Therefore, $\mu_{k}$ is of type $2^{\infty}$.

2. Suppose $f: I \rightarrow I$ is a continuous map with fixed points 0 and 1 . Define a map $\mu_{k} * f: I \rightarrow I$ such that

$$
\left.\mu_{k} * f\right|_{I-\left(a_{k}, b_{k}\right)}=\left.\mu_{k}\right|_{I-\left(a_{k}, b_{k}\right)}
$$

and

$$
\left.\mu_{k} * f\right|_{\left[a_{k}, b_{k}\right]}=\xi_{1,\left[c_{k}, d_{k}\right]} \circ f \circ \xi_{\left[a_{k}, b_{k}\right], I} .
$$

It is easy to see that the map $\mu_{k} * f$ is well defined and continuous and has fixed points 0 and 1 .

By definition,

$$
\mu_{k} * f\left(\left[a_{k}, b_{k}\right]\right)=\left[c_{k}, d_{k}\right] \text { and } \mu_{k} * f\left(\left[c_{k}, d_{k}\right]\right)=\left[a_{k}, b_{k}\right] .
$$

Hence, we have

(a) in $\left[a_{k}, b_{k}\right] \cup\left[c_{k}, d_{k}\right]$ there is no periodic point of $\mu_{k} * f$ with odd period. Obviously, the points 0,1 , and $e_{k}$ are fixed points of $\mu_{k} * f$. If $y \in\left(0, a_{k}\right)$, or if $y \in\left(d_{k}, 1\right)$, then $y$ is not a periodic point of $\mu_{k} * f$ because there is some $m>0$ such that $\left(\mu_{k} * f\right)^{m}(y) \in\left[a_{k}, d_{k}\right]$, and $\left[a_{k}, d_{k}\right]$ is an invariant subset of $\mu_{k} * f$. Similarly, if $y \in\left(b_{k}, c_{k}\right)$ and $y \neq e_{k}$, then $y$ is not a periodic point of $\mu_{k} * f$ because there is some $m>0$ such that $\left(\mu_{k} * f\right)^{m}(y) \in\left[a_{k}, b_{k}\right] \cup\left[c_{k}, d_{k}\right]$ and $\left[a_{k}, b_{k}\right] \cup\left[c_{k}, d_{k}\right]$ is an invariant subset of $\mu_{k} * f$. Thus,

(b) in $\left[0, a_{k}\right) \cup\left(b_{k}, c_{k}\right) \cup\left(d_{k}, 1\right]$ there are no periodic points of $\mu_{k} * f$ other than the fixed points 0,1 and $e_{k}$.

In addition,

$$
\begin{aligned}
\left.\left(\mu_{k} * f\right)^{2}\right|_{\left[a_{k}, b_{k}\right]} & =\left.\left.\mu_{k} * f\right|_{\left[c_{k}, d_{k}\right]} \circ \mu_{k} * f\right|_{\left[a_{k}, b_{k}\right]} \\
& =\left.\mu_{k}\right|_{\left[c_{k}, d_{k}\right]} \circ \xi_{I,\left[c_{k}, d_{k}\right]} \circ f \circ \xi_{\left[a_{k}, b_{k}\right], I} \\
& =\xi_{\left[c_{k}, d_{k}\right],\left[a_{k}, b_{k}\right]} \circ \xi_{I,\left[c_{k}, d_{k}\right]} \circ f \circ \xi_{\left[a_{k}, b_{k}\right], I} \\
& =\xi_{I,\left[a_{k}, b_{k}\right]} \circ f \circ \xi_{\left[a_{k}, b_{k}\right], I}
\end{aligned}
$$

From this, it follows that

(c) for any $m \geq 1$,

$$
\left.\left(\mu_{k} * f\right)^{2 m}\right|_{\left[a_{k}, b_{k}\right]}=\xi_{I,\left[a_{k}, b_{k}\right]} \circ f^{m} \circ \xi_{\left[a_{k}, b_{k}\right], I} .
$$

By statements (a), (b), and (c) one can prove the following proposition immediately.

Proposition 2. (1) If there is a periodic point of $\mu_{k} * f$ with period $n>1$, then $n$ is even and there is a periodic point of $f$ with period $n / 2$.

Therefore, if $f$ is of type $2^{\infty}$, then $\mu_{k} * f$ is of type $2^{\infty}$.

(2) If $x$ is a periodic point of $f$ with period $m>0$, then $y=\xi_{I,\left[a_{k}, b_{k}\right]}(x)$ is a periodic point of $\mu_{k} * f$ with period $2 m$; furthermore, if for some $u \in I$

$$
f^{l}(x)>u \quad \text { for } l=1,2, \ldots, m-1,
$$


then

$$
\left(\mu_{k} * f\right)^{l}(y)>\xi_{I,\left[a_{k}, b_{k}\right]}(u) \quad \text { for } l=1,2, \ldots, 2 m-1 .
$$

Proposition 3. Suppose that $f, f^{\prime}: I \rightarrow I$ are continuous maps with fixed points 0 and 1.

(1) If there is some $M>0$ such that

$$
\left|f(x)-f^{\prime}(x)\right|<M \quad \text { for all } x \in I,
$$

then

$$
\left|\mu_{k} * f(y)-\mu_{k} * f^{\prime}(y)\right|<M / 2^{k} \quad \text { for all } y \in I .
$$

(2) If $f$ and $f^{\prime}$ coincide on $I-(u, v)$, where $[u, v]$ is a subinterval of $I$, then $\mu_{k} * f$ and $\mu_{k} * f^{\prime}$ coincide on

$$
I-\left(\xi_{I,\left[a_{k}, b_{k}\right]}(u), \xi_{I,\left[a_{k}, b_{k}\right]}(v)\right) .
$$
then

PROOF. (1) If $y \in I-\left(a_{k}, b_{k}\right)$, then $\mu_{k} * f(y)-\mu_{k} * f^{\prime}(y)=0$, and if $y \in\left[a_{k}, b_{k}\right]$,

$$
\begin{aligned}
\mu_{k} * f(y)-\mu_{k} * f^{\prime}(y)= & \xi_{I,\left[c_{k}, d_{k}\right]}\left(f\left(\xi_{\left[a_{k}, b_{k}\right], I}(y)\right)\right) \\
& -\xi_{I,\left[c_{k}, d_{k}\right]}\left(f^{\prime}\left(\xi_{\left[a_{k}, b_{k}\right], I}(y)\right)\right) \\
= & \xi_{I,\left[c_{k}, d_{k}\right]}(f(x))-\xi_{I,\left[c_{k}, d_{k}\right]}\left(f^{\prime}(x)\right) \\
= & \left(d_{k}-c_{k}\right)\left(f(x)-f^{\prime}(x)\right) \\
= & \frac{1}{2^{k}}\left(f(x)-f^{\prime}(x)\right),
\end{aligned}
$$

where $x=\xi_{\left[a_{k}, b_{k}\right], I}(y)$. Hence for any $y \in I$,

$$
\left|\mu_{*} f(y)-\mu_{k} * f^{\prime}(y)\right|<M / 2^{k} .
$$

(2) By the definition, $\mu_{k} * f$ and $\mu_{k} * f^{\prime}$ coincide on $I-\left(a_{k}, b_{k}\right)$. Since $f$ and $f^{\prime}$ coincide on $I-(u, v), \mu_{k} * f$ and $\mu_{k} * f$ coincide on

$$
\begin{aligned}
\xi_{\left[a_{k}, b_{k}\right], I}^{-1}(I-(u, v)) & =\xi_{I,\left[a_{k}, b_{k}\right]}(I)-\xi_{I,\left[a_{k}, b_{k}\right]}((u, v)) \\
& =\left[a_{k}, b_{k}\right]-\left(\xi_{I,\left[a_{k}, b_{k}\right]}(u), \xi_{1,\left[a_{k}, b_{k}\right]}(v)\right) .
\end{aligned}
$$

Therefore, $\mu_{k} * f$ and $\mu_{k} * f^{\prime}$ coincide on

$$
I-\left(\xi_{I,\left[a_{k}, b_{k}\right]}(u), \xi_{I,\left[a_{k}, b_{k}\right]}(v)\right) .
$$

3. Let

$$
\begin{aligned}
\alpha_{3} & =\frac{1}{2^{3}} \\
\alpha_{4} & =\frac{1}{2^{3}}+\frac{1}{2} \cdot \frac{1}{2^{4}} \\
\alpha_{5} & =\frac{1}{2^{3}}+\frac{1}{2} \cdot \frac{1}{2^{4}}+\frac{1}{2} \cdot \frac{3}{4} \cdot \frac{1}{2^{5}} \\
& \ldots \ldots \\
\alpha_{k} & =\frac{1}{2^{3}}+\frac{1}{2} \cdot \frac{1}{2^{4}}+\frac{1}{2} \cdot \frac{3}{4} \cdot \frac{1}{2^{5}}+\cdots+\frac{1}{2} \cdot \frac{3}{4} \cdots \frac{2^{k-3}-1}{2^{k-3}} \cdot \frac{1}{2^{k}},
\end{aligned}
$$


and let

$$
\begin{aligned}
\beta_{3} & =1-\frac{3}{2^{3}} \\
\beta_{4} & =1-\frac{3}{2^{3}}-\frac{1}{2} \cdot \frac{3}{2^{4}} \\
\beta_{5} & =1-\frac{3}{2^{3}}-\frac{1}{2} \cdot \frac{3}{2^{4}}-\frac{1}{2} \cdot \frac{3}{4} \cdot \frac{3}{2^{5}} \\
& \ldots \ldots \ldots \cdots \\
\beta_{k} & =1-\frac{3}{2^{3}}-\frac{1}{2} \cdot \frac{3}{2^{4}}-\frac{1}{2} \cdot \frac{3}{4} \cdot \frac{3}{2^{5}}-\cdots-\frac{1}{2} \cdot \frac{3}{4} \cdots \frac{2^{k-3}-1}{2^{k-3}} \cdot \frac{3}{2^{k}}
\end{aligned}
$$

Obviously, $\alpha_{3}<\alpha_{4}<\alpha_{5}<\cdots<\alpha_{k-1}<\alpha_{k}<\cdots$ and $\beta_{3}>\beta_{4}>\beta_{5}>\cdots>$ $\beta_{k-1}>\beta_{k}>\cdots$.

Since both sequences $\alpha_{3}, \alpha_{4}, \ldots$ and $\beta_{3}, \beta_{4}, \ldots$ are monotone and bounded, their limits exist. Let $\alpha=\lim _{k \rightarrow \infty} \alpha_{k}$ and $\beta=\lim _{k \rightarrow \infty} \beta_{k}$. By a simple calculation, we have that

$$
\beta_{k}-\alpha_{k}=\frac{1}{2} \cdot \frac{3}{4} \cdots \frac{2^{k-2}-1}{2^{k-2}} .
$$

Thus,

$$
\beta-\alpha=\lim _{k \rightarrow \infty}\left(\beta_{k}-\alpha_{k}\right)=\prod_{i=1}^{\infty}\left(1-\frac{1}{2^{i}}\right)>0
$$

(see [4]). Hence,

$(*)$

$$
\alpha_{3}<\alpha_{4}<\cdots<\alpha_{k-1}<\alpha_{k}<\cdots<\alpha<\beta<\cdots<\beta_{k}<\beta_{k-1}<\cdots<\beta_{4}<\beta_{3} .
$$

By the definition of the notation, $\xi_{I,[u, v]}, \xi_{I,[u, v]}(x)=u+(v-u) x$ for any $x \in I$. Therefore,

$$
\begin{aligned}
\xi_{I,\left[a_{3}, b_{3}\right]} \circ \xi_{I,\left[a_{4}, b_{4}\right]} \circ \cdots \circ \xi_{I,\left[a_{k-1}, b_{k-1}\right]}\left(a_{k}\right) \\
\quad=a_{3}+\left(b_{3}-a_{3}\right) \xi_{I,\left[a_{4}, b_{4}\right]} \circ \cdots \circ \xi_{I,\left[a_{k-1}, b_{k-1}\right]}\left(a_{k}\right) \\
\quad=\ldots \\
\quad=a_{3}+\left(b_{3}-a_{3}\right) a_{4}+\cdots+\left(b_{3}-a_{3}\right)\left(b_{4}-a_{4}\right) \cdots\left(b_{k-1}-a_{k-1}\right) a_{k} \\
\quad=\frac{1}{2^{3}}+\frac{1}{2} \cdot \frac{1}{2^{4}}+\cdots+\frac{1}{2} \cdot \frac{3}{4} \cdots \frac{2^{k-3}-1}{2^{k-3}} \cdot \frac{1}{2^{k}} \\
\quad=\alpha_{k} .
\end{aligned}
$$

In brief,

$(* *)_{1}$

$$
\alpha_{k}=\xi_{I,\left[a_{3}, b_{3}\right]} \circ \xi_{I,\left[a_{4}, b_{4}\right]} \circ \cdots \circ \xi_{I,\left[a_{k-1}, b_{k-1}\right]}\left(a_{k}\right),
$$

and by a similar calculation,

$$
(* *)_{2} \quad \beta_{k}=\xi_{I,\left[a_{3}, b_{3}\right]} \circ \xi_{I,\left[a_{4}, b_{4}\right]} \circ \cdots \circ \xi_{I,\left[a_{k-1}, b_{k-1}\right]}\left(b_{k}\right) .
$$


4. Now, we define

$$
\begin{aligned}
\chi_{3} & =\mu_{3} \\
\chi_{4} & =\mu_{3} * \mu_{4} \\
\chi_{5} & =\mu_{3} *\left(\mu_{4} * \mu_{5}\right), \\
& \ldots \ldots \\
\chi_{k} & =\mu_{3} *\left(\mu_{4} *\left(\cdots *\left(\mu_{k-1} * \mu_{k}\right) \cdots\right)\right), \\
& \ldots \ldots \ldots
\end{aligned}
$$

PROPOSITION 4. (1) $\chi_{k}$ is of type $2^{\infty}$.

(2) $\left.\chi_{k+1}\right|_{I-\left(\alpha_{k}, \beta_{k}\right)}=\left.\chi_{k}\right|_{I-\left(\alpha_{k}, \beta_{k}\right)}$.

Therefore, if $l \geq k$, then

$$
\left.\chi_{k}\right|_{I-\left(\alpha_{k}, \beta_{k}\right)}=\left.\chi_{l}\right|_{I-\left(\alpha_{k}, \beta_{k}\right)} .
$$

(3) $\alpha_{k}$ is a periodic point of $\chi_{k}$ with period $2^{k-2}$, and

$$
\left(\chi_{k}\right)^{m}\left(\alpha_{k}\right)>\beta_{k} \quad \text { for } m=1,2, \ldots, 2^{k-2}-1 \text {. }
$$

Therefore, if $l \geq k$, then $\alpha_{k}$ is a periodic point of $\chi_{l}$ with period $2^{k-2}$, and

$$
\left(\chi_{l}\right)^{m}\left(\alpha_{k}\right)>\beta_{k} \quad \text { for } m=1,2, \ldots, 2^{k-2}-1 .
$$

(4) For any $x \in I$,

$$
\left|\chi_{k+1}(x)-\chi_{k}(x)\right|<2^{-(k+3)(k-2) / 2} .
$$

PrOOF. (1) Propositions 1 and 2(1) imply the statement (1) of this proposition.

(2) By the definition, $\mu_{k} * \mu_{k+1}$ and $\mu_{k}$ coincide on $I-\left(a_{k}, b_{k}\right)$. By Proposition $3(2), \mu_{k-1} *\left(\mu_{k} * \mu_{K+1}\right)$ and $\mu_{k-1} * \mu_{k}$ coincide on

$$
I-\left(\xi_{I,\left[a_{k-1}, b_{k-1}\right]}\left(a_{k}\right), \xi_{I,\left[a_{k-1}, b_{k-1}\right]}\left(b_{k}\right)\right) .
$$

Applying Proposition 3(2) repeatedly, we have that $\chi_{k+1}$ and $\chi_{k}$ coincide on

$$
\begin{array}{r}
I-\left(\xi_{I,\left[a_{3}, b_{3}\right]} \circ \xi_{I,\left[a_{4}, b_{4}\right]} \circ \cdots \circ \xi_{I-\left[a_{k-1}, b_{k-1}\right]}\left(a_{k}\right),\right. \\
\left.\xi_{I,\left[a_{3}, b_{3}\right]} \circ \xi_{I,\left[a_{4}, b_{4}\right]} \circ \cdots \circ \xi_{I,\left[a_{k-1}, b_{k-1}\right]}\left(b_{k}\right)\right),
\end{array}
$$

which is $I-\left(\alpha_{k}, \beta_{k}\right)$ by $(* *)_{1}$ and $(* *)_{2}$.

(3) $a_{k}$ is a periodic point of $\mu_{k}$ with period 2 and $\mu_{k}\left(a_{k}\right)=c_{k}>b_{k}$. By Proposition 2(2), $\xi_{I,\left[a_{k-1}, b_{k-1}\right]}\left(a_{k}\right)$ is a periodic point of $\mu_{k-1} * \mu_{k}$ with period 4, and

$$
\left(\mu_{k-1} * \mu_{K}\right)^{m}\left(\xi_{I,\left[a_{k-1}, b_{k-1}\right]}\left(a_{k}\right)\right)>\xi_{I,\left[a_{k-1}, b_{k-1}\right]}\left(b_{k}\right)
$$

for $m=1,2,3$.

Applying Proposition 2(2) repeatedly, we have $\alpha_{k}=\xi_{I,\left[a_{3}, b_{3}\right]} \circ \xi_{I,\left[a_{4}, b_{4}\right]} \circ \cdots \circ$ $\xi_{I,\left[a_{k-1}, b_{k-1}\right]}\left(a_{k}\right)$ is a periodic point of $\chi_{k}$ with a period of $2^{k-2}$, and

$$
\left(\chi_{k}\right)^{m}\left(\alpha_{k}\right)>\xi_{I,\left[a_{3}, b_{3}\right]} \circ \xi_{I,\left[a_{4}, b_{4}\right]} \circ \cdots \circ \xi_{I,\left[a_{k-1}, b_{k-1}\right]}\left(b_{k}\right)=\beta_{k}
$$

for $m=1,2, \ldots, 2^{k-2}-1$.

(4) By the definition, for any $x \in I$

$$
\left|\mu_{k} * \mu_{k+1}(x)-\mu_{k}(x)\right|<2^{-k} \text {. }
$$


By Proposition 3(1), for any $x \in I$

$$
\left|\mu_{k-1} *\left(\mu_{k} * \mu_{k+1}\right)(x)-\mu_{k-1} * \mu_{k}(x)\right|<2^{-(k-1)} \cdot 2^{-k} \text {. }
$$

Applying Proposition 3(1) repeatedly, we have that for any $x \in I$

$$
\begin{aligned}
\left|\chi_{k+1}(x)-\chi_{k}(x)\right|= & \mid \mu_{3} *\left(\mu_{4} *\left(\cdots *\left(\mu_{k} * \mu_{k+1}\right) \cdots\right)\right)(x) \\
& -\mu_{3} *\left(\mu_{k} *\left(\cdots *\left(\mu_{k-1} * \mu_{k}\right) \cdots\right)\right)(x) \mid \\
< & 2^{-3} \cdot 2^{-4} \cdots 2^{-k} \\
= & 2^{-(k+3)(k-2) / 2} .
\end{aligned}
$$

5. By Proposition 4(4) the sequence of the maps $\chi_{3}, \chi_{4}, \ldots$ is uniformly convergent, and its limit map, denoted by $\chi_{\infty}$, is continuous. By a theorem of Block (see Main Theorem in [3]), $\chi_{\infty}$ is of type $2^{\infty}$ because all $\chi_{k}$ 's are of type $2^{\infty}$.

Now, we will state and prove our main conclusion as follows.

THEOREM. The set of recurrent points of the continuous map $\chi_{\infty}: I \rightarrow I$, which is of type $2^{\infty}$, is not closed.

ProOF. By Proposition 4(2) and the definition of $\chi_{\infty}$ it is easy to see that

$$
\left.\chi_{\infty}\right|_{I-\left(\alpha_{k}, \beta_{k}\right)}=\left.\chi_{k}\right|_{I-\left(\alpha_{k}, \beta_{k}\right)} .
$$

Hence, by Proposition 4(3), $\alpha_{k}$ is periodic of $\chi^{\infty}$ with period $2^{k-2}$, and

$$
\chi_{\infty}^{m}\left(\alpha_{k}\right)>\beta_{k}>\beta \text { for } m=1,2, \ldots, 2^{k-2}-1 .
$$

Consider the accumulation point $\alpha=\lim _{k \rightarrow \infty} \alpha_{k}$ of periodic points of $\chi_{\infty}$. For any positive integer $m>0$

$$
\chi_{\infty}^{m}(\alpha)=\lim _{k \rightarrow \infty} \chi_{\infty}^{m}\left(\alpha_{k}\right)
$$

Thus, $\chi_{\infty}^{m}>\beta$ because $\chi_{\infty}^{m}\left(\alpha_{m+2}\right)>\beta, \chi_{\infty}^{m}\left(\alpha_{m+3}\right)>\beta, \ldots$ Since $\alpha<\beta$ (see $(*)$ ), $\alpha$ is not a recurrent point of $\chi_{\infty}$. This shows that the set of recurrent points is not closed.

\section{REFERENCES}

1. Xiong Jincheng, The periods of periodic points of continuous self-maps of the interval whose recurrent points form a closed set, J. China Univ. Sci. Tech. 13 (1983), 134-135.

2. A. M. Bloh, The asymptotic behaviour of one-dimensional dynamical systems, Uspekhi Mat. Nauk 37 (1982), 175-176.

3. L. Block, Stability of periodic orbits in the theorem of Sarkovski, Proc. Amer. Math. Soc. 81 (1981), 335-336.

4. A. Weil, Two lectures on number theory, past and present, Enseign. Math. 20 (1974), 87-110.

DePARTMENT OF MATHEMATICS, UNIVERsity of MARYland, College PARK, MARYLAND 20742

International Centre for Theoretical Physics, Trieste, Italy 